Western University

Scholarship@Western

$8-1-2015$

Contrasting styles in cognition and behaviour in bumblebees and honeybees.

David F Sherry

Caroline G Strang

Follow this and additional works at: https://ir.lib.uwo.ca/psychologypub

Part of the Biology Commons, and the Psychology Commons

Citation of this paper:

Sherry, David F and Strang, Caroline G, "Contrasting styles in cognition and behaviour in bumblebees and honeybees." (2015). Psychology Publications. 177.

https://ir.lib.uwo.ca/psychologypub/177 


\title{
Contrasting styles in cognition and behaviour in bumblebees and honeybees
}

\author{
David F. Sherry*, Caroline G. Strang \\ Department of Psychology, University of Western Ontario, London, ON, Canada N6A 5C2
}

\section{A R T I C L E I N F O}

\section{Article history:}

Received 25 April 2014

Received in revised form 2 September 2014

Accepted 2 September 2014

Available online 10 September 2014

\section{Keywords:}

Bumblebee

Honeybee

Memory

Numerosity

Orientation

Timing

\begin{abstract}
A B S T R A C T
Bumblebees and honeybees have been the subjects of a great deal of recent research in animal cognition. Many of the major topics in cognition, including memory, attention, concept learning, numerosity, spatial cognition, timing, social learning, and metacognition have been examined in bumblebees, honeybees, or both. Although bumblebees and honeybees are very closely related, they also differ in important ways, including social organization, development, and foraging behaviour. We examine whether differences between bumblebees and honeybees in cognitive processes are related to differences in their natural history and behaviour. There are differences in some cognitive traits, such as serial reversal learning and matching-to-sample, that appear related to differences between bumblebees and honeybees in foraging and social behaviour. Other cognitive processes, such as numerosity, appear to be very similar. Despite the wealth of information that is available on some aspects of bumblebee and honeybee cognition and behaviour, there are relatively few instances, however, in which adequate data exist to make direct comparisons. We highlight a number of phenomena, including concept learning, spatial cognition, timing, and metacognition, for which targeted comparative research may reveal unexpected adaptive variation in cognitive processes in these complex animals. This article is part of a Special Issue entitled: In Honor of Jerry Hogan.
\end{abstract}

(C) 2014 Elsevier B.V. All rights reserved.
A recurring theme in Jerry Hogan's writing and teaching is the distinction between cause and function in the study of animal behaviour (Hogan and Bolhuis, 2009; Hogan, 1994). As Jerry points out in his chapter in this issue, the relation between cause and function in animal behaviour is complex (Hogan, in press). Functional explanations of behaviour cannot serve the same role as causal explanations, and much fruitless argument and debate can result when intentionally or unintentionally functional accounts are made to do the explanatory work of causal accounts. Functional outcomes cannot make behaviour happen. At the same time, research on the function of behaviour, such as the optimization of nectar collection by pollinators, can raise causal questions about the cognitive processes that make an outcome possible. Similarly, a clear understanding of causal mechanisms can explain why expected functional outcomes sometimes do not occur. Causal constraints on how memory or perception work may impose limits on

\footnotetext{
(5) Cause and function in behavioural biology-A tribute to the contributions of Jerry A. Hogan Behavioural Processes 2014 Special Issue (J.J. Bolhuis and L.-A. Giraldeau, Editors).

* Corresponding author. Tel.: +1 5196612111.

E-mail address: sherry@uwo.ca (D.F. Sherry).
}

what a pollinator can achieve. On an evolutionary time scale, natural selection can modify causal mechanisms such as memory or perception and produce adaptively specialized causal mechanisms that may be difficult to understand without knowing their function. In this paper, we will try to steer a course between functional and causal accounts of animal cognition and ask whether the functions of bumblebee and honeybee behaviour can help us analyze causal properties of cognition in these closely related but very different social insects.

The comparative study of animal cognition also focuses on recurring themes. Memory, attention, concept learning, numerosity, spatial cognition, timing, social learning, and metacognition are standard topics in texts and reference books of animal cognition (Shettleworth, 2013; Roberts, 1998; Zentall and Wasserman, 2012). Perhaps surprisingly, all of these topics and more have been investigated in bumblebees and honeybees. We will describe some recent discoveries about bee cognition that highlight the potential of bumblebees and honeybees for examining challenging questions about the causal organization of animal cognition.

There is a vast amount of research on the mechanisms of learning, memory and cognition in honeybees and rather less on bumblebees. Much of the research on honeybees focuses, either directly or indirectly, on their dance language and on how the 


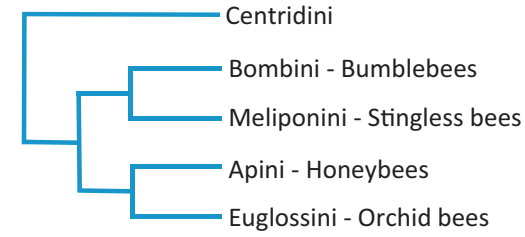

Fig. 1. Phylogeny of the bumblebees and honeybees based on analyses of molecular data (Kawakita et al., 2008; Cardinal et al., 2010). Bombini, Meliponini, Apini and Euglossini make up the monophyletic corbiculate group. The Centridini are a noncorbiculate sister group of bees within the subfamily Apinae. Adapted from Cardinal et al. (2010).

direction and distance information conveyed by the waggle dance is put to use: how the sun's azimuth and skylight polarization are used to determine compass direction (e.g. Evangelista et al., 2014), and how distance and position are estimated (Cheng, 2000). There has been, in addition, a great deal of research on the molecular mechanisms of learning and memory in honeybees using the proboscis extension response (PER) as a model system. We will not attempt to review this large body of honeybee research here. Comprehensive recent reviews are available (Menzel, 2012; Giurfa and Sandoz, 2012; Srinivasan, 2011; Couvillon, 2012; Grüter and Farina, 2009). Research on learning, memory, and cognition in bumblebees, in contrast, tends to focus on foraging decisions and how bumblebees exploit floral nectar and pollen resources. Trapline foraging, the use of space, floral constancy, and the coevolution of bumblebee behaviour and floral phenotypes are recurring themes (Heinrich, 1979; Goulson, 2009; Kearns and Thomson, 2001; Chittka et al., 1999; Lihoreau et al., 2010). Our goals in this paper are to examine a few areas where there appear to be interesting differences in cognition between honeybees and bumblebees and to relate these where possible to differences in honeybee and bumblebee behaviour and natural history.

\section{Bumblebees and honeybees}

There are about 20,000 species of bees, distributed over seven families (Danforth et al., 2013). Bumblebees and honeybees are members of the same family, Apidae, and the same subfamily Apinae. Bumblebees belong to the tribe Bombini and all belong to a single genus, Bombus, while honeybees are in the tribe Apini that likewise comprises a single genus, Apis. Though not each other's closest phylogenetic relatives, the bumblebees and honeybees are nevertheless very closely related (Hedtke et al., 2013; Danforth et al., 2013; Cardinal and Danforth, 2011; Thompson and Oldroyd, 2004). The nearest relatives of bumblebees are the stingless bees in the tribe Meliponini while the nearest relatives of the honeybees are the tribe Euglossini, the orchid bees (Fig. 1).

These four tribes (Bombini and Meliponini, Apini and Euglossini) are known as the corbiculate bees for the corbicula or pollen baskets on the hind legs used for carrying pollen. There are 7 species of Apis worldwide and about 250 species of Bombus.

Although most species of bees are solitary, both bumblebees and honeybees are eusocial, probably deriving their eusociality from a primitively eusocial common ancestor of the corbiculate bees (Cardinal and Danforth, 2011). Bumblebees are considered primitively eusocial because queen and worker bumblebees differ primarily in size, in contrast to honeybees which show greater morphological caste specialization, and because new bumblebee nests are established by a single foundress queen whereas new honeybee hives are formed by swarms that include a queen and many workers (Cardinal and Danforth, 2011). Both bumblebees and honeybees collect floral nectar and pollen, and honeybees also collect propolis which they use to seal and reinforce the hive. Both bumblebees and honeybees are important pollinators of wild plants and commercial crops. Both are polylectic - they collect pollen from many different unrelated types of plants - in contrast to many bees that collect pollen from only one or a few species of plants, though there is at least one monolectic bumblebee, Bombus consobrinus, a specialist on monkshood Aconitum (Laverty and Plowright, 1988). Most of what is known about learning, memory, and cognition in honeybees comes from research on the domestic honeybee Apis mellifera while research on bumblebees examines more species. Increasingly, however, research on bumblebees focuses on two species that are raised commercially for pollination of greenhouse crops, Bombus impatiens in North America and Bombus terrestris in Europe.

Bumblebees and honeybees differ in many ways that may affect cognitive processes or how cognition is deployed to solve problems bees encounter in the wild. Bumblebee colonies are smaller than honeybee colonies, consisting of several hundred individuals compared to many thousands of individuals in a honeybee colony. This means that the behaviour of an individual bumblebee has a greater effect on the success of the colony than does the behaviour of an individual honeybee. Honeybees originated in the tropics and bumblebees in northern temperate regions (Michener, 2007). Bumblebees are typically larger than honeybees. A single bumblebee is energetically more costly to its colony than a single honeybee, but bumblebees are less costly in metabolic rate per unit of mass (Townsend-Mehler et al., 2011): it takes fewer calories to fuel a gram of bumblebees than a gram of honeybees.

Honeybees have better colour discrimination than bumblebees, but bumblebees show greater acuity in the detection of colour stimuli than honeybees (Dyer et al., 2008). It is possible that the temperate zone origin of bumblebees placed a greater selective advantage on the detection of small colour targets, namely dispersed patches of flowers, than the tropical zone origin of honeybees in which forest trees present thousands of flowers in a highly localized concentration (Dyer et al., 2008). Bumblebees and honeybees also differ in attentional processes. Bumblebees show slow but accurate parallel search compared to the fast but less accurate serial search of honeybees when searching for a target in the presence of distractors (Morawetz and Spaethe, 2012).

The division of labour in the colony differs between honeybees and bumblebees. In honeybees, workers progress though various nest and foraging tasks in an age-dependent fashion. In bumblebees workers of all ages and sizes may perform nest or foraging duties. Larger bumblebees bring nectar to the colony at a higher rate (Spaethe and Weidenmüller, 2002) but individuals of all sizes forage for nectar and pollen. Within a bumblebee colony some individuals have spatially small areas of activity, usually associated with feeding larvae, while others move in spatially larger areas foraging, fanning, or guarding the nest. The duties are neither age-dependent as in honeybees nor size dependent (Jandt and Dornhaus, 2009).

Honeybee foragers communicate to each other about sites where nectar and pollen are available using the well-known dance language, bumblebees do not. Bumblebees do, however, obtain information that floral nectar sources are available from the "excited runs" and pheromone signals of foragers returning to the nest and obtain olfactory information in the nest about what kinds of nectar sources these are (Dornhaus and Chittka, 2001, 2004, 2005; Saleh and Chittka, 2006; Molet et al., 2009). Foraging bumblebees also learn from interactions at flowers with other bumblebees to prefer certain flowers (Worden and Papaj, 2005; Leadbeater and Chittka, 2005; Avarguès-Weber and Chittka, 2014) and which flowers have a reduced risk of predation (Dawson and Chittka, 2014). Bumblebees are avid nectar robbers, cutting into the corolla of some flowers to obtain nectar, and the presence of these cut corollas promotes nectar robbing in naïve bees (Leadbeater and Chittka, 2008; Goulson et al., 2013). Although Darwin thought that honeybees could learn to exploit cuts made in corollas by bumblebees 
(Darwin, 1841; Romanes, 1884), there appears to be no contemporary research on social learning of this kind by honeybees.

Bumblebee pollen loads from a single foraging trip often contain more different types of pollen than is the case for honeybees, indicating that they visit a greater variety of flowers per foraging trip, and the pollen that is collected by bumblebees is higher in protein and essential amino acid content than pollen collected by honeybees (Leonhardt and Blüthgen, 2012) suggesting that bumblebees may be better able to perceive aspects of pollen quality than honeybees.

B. impatiens abandons a high value food source sooner than $A$. mellifera when the concentration of nectar declines (TownsendMehler et al., 2011). Bumblebees also, however, return to that nectar source more readily than honeybees do. Furthermore, bumblebees are more likely than honeybees to search for and successfully discover alternative nectar sources following a drop in nectar concentration at the source they are feeding from. Townsend-Mehler and Dyer (2012) found that honeybees are more resistant to extinction at a high value food source, that is, they perseverate after bumblebees have given up and moved on. Bumblebees are more influenced by their history of reward with a high value food source than honeybees are, and show a smaller negative contrast effect when provided with a non-preferred low quality source of nectar (Townsend-Mehler and Dyer, 2012). There are differences, however, between species of Bombus in floral constancy the tendency to visit one type of flower exclusively - indicating that differences between Bombus and Apis in the likelihood of switching among food sources may not be absolute (Raine et al., 2006).

The extraordinary behavioural complexity of honeybees and bumblebees, both as individuals and as an integrated functioning colony, is familiar to most students of animal behaviour. We will try to assess whether there are consistent differences in bumblebee and honeybee cognition that transcend experimental procedures and paradigms, and ask whether it is possible to account for these differences in terms of bumblebee and honeybee behaviour and natural history. As Jerry Hogan would more accurately put it, are there differences between bumblebees and honeybees in the function of their behaviour that are correlated with differences in causal cognitive mechanisms?

\section{Memory}

Two very simple but widely used procedures in research on animal cognition are serial reversal and matching-to-sample. The former requires that an animal learn to discriminate between stimuli and then change what it has learned. The latter requires an animal to retain the memory of a sample stimulus, usually briefly, and then show by a choice whether it can match that memory to stimuli presented subsequently. Both procedures have been used to examine memory in bumblebees and honeybees.

\subsection{Serial reversal learning}

Reversal learning requires animals to learn an initial discrimination between rewarded and unrewarded stimuli, recognize when the reward contingency is changed, and respond accordingly (Shettleworth, 1998). In serial reversal learning, the change in reward contingency is repeated multiple times, requiring the animal to continually change its behaviour. Serial reversal learning is widely used in research on animal cognition because it serves as a measure of behavioural flexibility, can be adapted to test almost any species, and provides information about how an animal solves the reversal task (Bitterman, 1969). If the animal reaches perfect performance in a reversal task, switching after only a single error, it suggests a win-stay/lose-shift rule is being used (Shettleworth,
1998). If performance improves but falls short of single trial reversal, it suggests the animal has not learned a rule for switching, but is burdened by proactive interference and is therefore less likely to correctly switch back to a previously rewarded stimulus (Bitterman, 1969). These properties of serial reversal learning have led to its use in research on cognition in many species (Bitterman, 1969; Davey, 1989; Shettleworth, 2010a,b).

Differences in natural foraging behaviour between bumblebees and honeybees are considerable and suggest there may be differences in behavioural flexibility between them. Floral constancy is the tendency for an individual bee, once it has begun to forage, to concentrate on a single species of plant almost exclusively and both honeybees and bumblebees tend to be florally constant (Free, 1963; Free, 1970). As noted earlier, however, bumblebees may be both quicker to abandon a depleting nectar source and quicker to return to it whereas honeybees tend to perseverate on such a depleting resource (Townsend-Mehler et al., 2011). This may occur because honeybee foragers are recruited by the dance language to highly rewarding flowers (Seeley, 1985), and can locate flowers and forage productively without having to determine by trial and error which flowers are rewarding and which are not. This is not the case for bumblebees which usually sample many different flower species before becoming constant (Heinrich et al., 1977). If the natural foraging behaviour of honeybees does not require them to vary the types of flowers that they visit, while the natural foraging behaviour of bumblebees requires visiting both rewarding and non-rewarding flowers, it is possible that there are differences in behavioural flexibility detectable by differences in serial reversal learning.

In honeybees serial reversal has been studied in harnessed and in free flying bees. Harnessed bees are tested using the proboscis extension reflex (PER) in which a bee is conditioned to extend its proboscis when an $\mathrm{S}+$ odour is administered to its antennae (the olfactory organ of bees) and refrain from responding to an S- odour (Bitterman et al., 1983). Mota and Giurfa (2010) tested serial reversal learning in honeybees using PER and found that bees given three reversals of five trials per reversal significantly changed their responding following each reversal. The extent to which they changed their responding decreased, however, with each reversal. This indicates that, contrary to what is typically found in animals tested on serial reversal, honeybees show worse performance with repeated reversals and, in fact, in this experiment they eventually lost the ability to discriminate between the S+ and S- stimuli.

This result closely resembles results with free flying honeybees tested on a similar number of colour reversals over a similar number of trials (von Helversen, 1974; Couvillon and Bitterman, 1986). When free flying honeybees were tested for more trials ( 8 or 10 per reversal), however, they did not gradually generalize responding to both stimuli, but were able to reverse their choice of the S+ stimulus and showed a similar pattern of discrimination over all reversals trials (von Helversen, 1974; Couvillon and Bitterman, 1986). This suggests that the inability of the bees to adapt to the reversals in the PER procedure may be due to the small number of trials rather than the inability of honeybees to adapt to reversal at all.

Dyer et al. (2014) subjected free-flying honeybees to three reversals of a two-colour discrimination. The mean performance of the group of 32 bees was $50 \%$ correct by the third reversal. Analysis of the behaviour of individual bees showed that 4 reversed their colour preference when the reward contingency reversed, 4 maintained their original colour preference across all reversals, and 24 bees behaved in a way that Dyer et al. (2014) called "Ficklecircumspect". As in the study by Mota and Giurfa (2010), successive reversals led to mean performance at the chance level.

A further study of reversal learning in honeybees compared bees' PER responses on a reversal task when prior training had included either reversals or an equivalent amount of training 
without reversals (Komischke et al., 2002). Honeybees that had experienced reversals changed their pattern of responding on the final reversal more than those that had the same number of training trials without any reversals. Neither group, however, managed to completely reverse their responding on the final reversal and respond to the newly rewarded stimulus more than to the previously rewarded stimulus. Nonetheless, prior experience did seem to facilitate reversal learning, supporting the conclusion that previous failures of honeybees on reversal tasks could be due to training conditions.

In general, the results of serial reversal tests on honeybees under a variety of conditions show worsening performance with an increasing number of reversals. Results with bumblebees have been very different, showing improvement in reversal learning (Chittka, 1998; Strang and Sherry, 2014). Chittka (1998) trained bumblebees to turn either left or right in a T-maze - indicated by a centrally located colour cue - in order to receive a sucrose reward. A single bumblebee was then trained on 11 reversals, one reversal occurring each day, and given 100 trials on each reversal. The bee's accuracy declined dramatically at the start of the reversals, but then improved until by the eighth reversal, the bee made only one error in the first eight post reversal trials.

In an experiment involving a single reversal of a blue/yellow colour discrimination, bumblebees quickly reversed their colour preference (Raine and Chittka, 2012). Speed of learning the initial discrimination was correlated with overnight retention of the discrimination and with speed of learning the reversed discrimination on the following day.

We tested bumblebees on nine reversals of 40 trials each and found significant improvement across reversals (Strang and Sherry, 2014). The number of errors bees made in the first block of trials immediately following a reversal decreased across reversals, and the number of errors made in subsequent blocks, when the reward contingency was stable, fell to minimal levels (Fig. 2). The bumblebees learned to reverse. Their repeated experience with serial reversal improved their ability to reverse, in contrast to results with honeybees that tend to show a deteriorating ability to reverse with repeated reversals. These two studies clearly show that bumblebees are behaviourally flexible, in contrast to results with honeybees that indicate a breakdown in discrimination under rapidly changing reward conditions.

Bumblebees may thus differ from honeybees in behavioural flexibility in a way that is correlated with their usual foraging behaviour. Whether this difference is the result of evolutionary modification of learning, the result of individual experience, or an outcome of events during bumblebee and honeybee development remains to be determined.

\subsection{Matching-to-sample}

Cognitive processes more complex than reversal learning, such as concept learning, numerosity, and metacognition, have increasingly been explored in bees. There has been research with both bumblebees and honeybees, but honeybees have been the preferred subjects, perhaps reflecting honeybee researchers' focus on the mechanisms of learning and memory. The preference for honeybees could also be due, however, to their aptitude at solving matching-to-sample (MTS) tasks (Giurfa et al., 2001; Zhang et al., 2005; Zhang and Pahl, 2012; Gross et al., 2009). In a matchingto-sample task an animal is presented with a stimulus and then presented with two choice stimuli, one of which is a match to the sample stimulus and is rewarded while the other is a non-match and is not rewarded (Shettleworth, 1998, 2010). A variable delay can be introduced between the sample and the choice, turning the task into delayed matching-to-sample (DMTS). The task can also be varied to provide a reward for choosing the non-matching
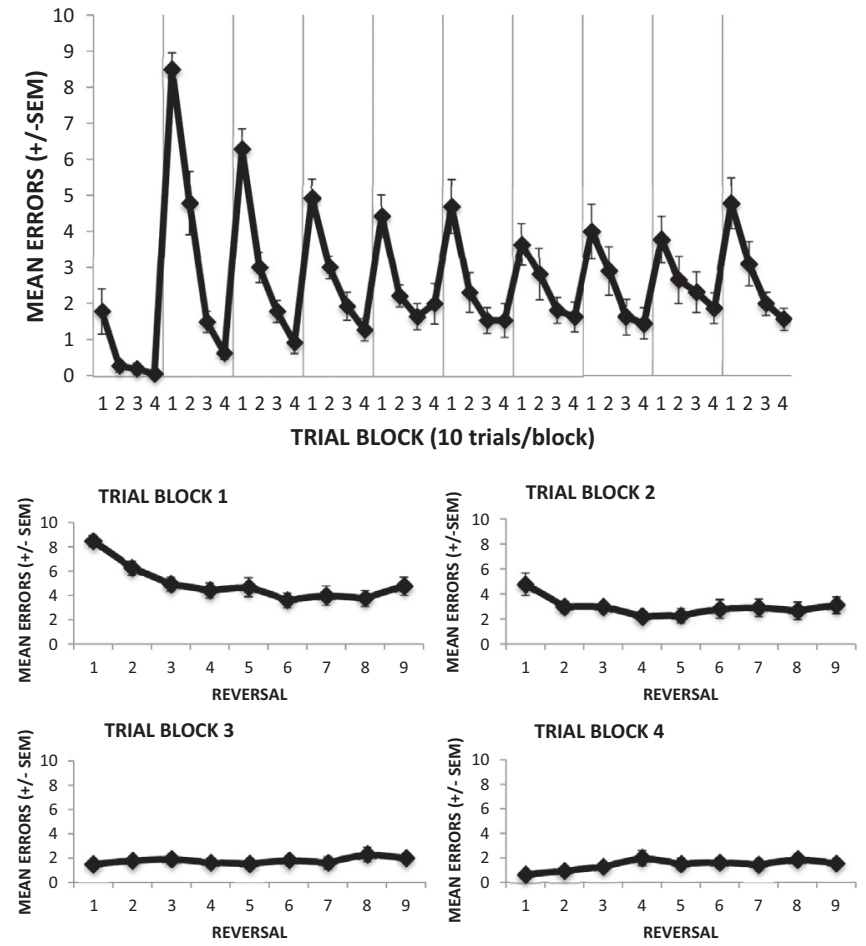

Fig. 2. Serial reversal learning in bumblebees. Free flying bees were rewarded with sucrose for a yellow/blue colour discrimination. The reward contingency was reversed after every four blocks of trials, shown by the grey vertical lines (upper panel). The number of errors immediately following reversal decreased across reversals and decreased to minimal levels by the final trial block within a session (lower panel). Reprinted with permission from Strang and Sherry (2014).

stimulus, making the task non-matching-to-sample. The relationship between the sample and choice stimuli can be symbolic rather than a literal match. A blue sample, for instance, might require choosing a vertical but not a horizontal line for a correct match in a symbolic matching-to-sample task. MTS tasks have been used extensively to study not only animal memory (Blough, 1959), but also concept learning (Zentall and Hogan, 1978; Wright, 1997) and metacognition (Hampton, 2001). The versatility of MTS makes many questions about cognition accessible once an animal has learned the basic task.

There are numerous studies in which honeybees have successfully learned DMTS tasks (Giurfa et al., 2001; Zhang et al., 2005; Gross et al., 2009; Zhang and Pahl, 2012). In the standard paradigm, a honeybee flies into a Y-maze and is presented with a sample. After inspecting the sample, the bee flies through a baffle into a choice chamber where it is presented with matching and non-matching stimuli. The bee makes a choice by flying into the reward chamber through the opening indicated by the matching stimulus. Although the task is different from the touch screen or key peck apparatus used with laboratory rats and pigeons, honeybees show the typical memory decline with increasing delays between the sample and the choice stimuli, indicating that successful performance depends on working memory as in other DMTS designs (Zhang et al., 2005; Zhang and Pahl, 2012).

In contrast to the relative abundance of honeybee DMTS studies there is a conspicuous absence of similar work in bumblebees. It is possible there are few results on DMTS with bumblebees because there have been few attempts. It is also possible there have been unsuccessful attempts to train bumblebee on DMTS. As Perry et al. (2013) point out, unsuccessful attempts to detect learning phenomena in invertebrates can be informative. We attempted to train bumblebees on a matching-to-sample task comparable to those 


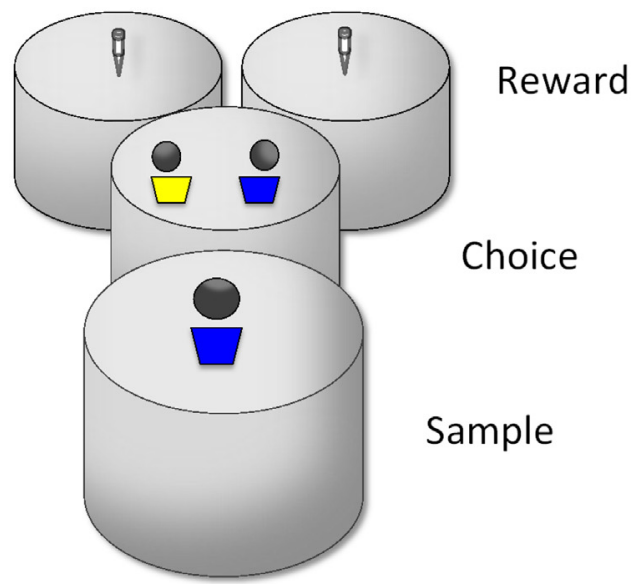

win-stay foraging strategy (Collett, 2005). As described previously in connection with reversal learning, honeybees show greater flower constancy than bumblebees (Free, 1963, 1970). If success on the DMTS task is the outcome of a win-stay strategy, then the observed species difference in matching-to-sample may be due to a difference in floral constancy. Honeybees may tend to pick a preferred target stimulus and stick with it, despite the reduced reward rate they experience.

The natural win-stay strategy may also explain honeybees' success on the non-matching version of the task. Collett (2005) suggested that in a non-matching task, pairing the matching stimulus with the aversive outcome of non-reward biases honeybees away from the sample. When the bee next encounters the sample stimulus and associated contextual cues, it may be biased away from that stimulus and successfully choose the non-match. This mechanism may account for both the impressive success of honeybees on DMTS tasks and the failure of bumblebees, and may be a much simpler explanation of the bees' behaviour than supposing that bees learn a "concept" of match and non-match (Chittka and Jensen, 2011).

Although use of a win-stay strategy can account for success on the standard DMTS task, it cannot account for success on symbolic versions of the task (SDMTS). Zhang et al. (1999) successfully trained honeybees on a symbolic matching task in which a bee had to navigate a Y-maze by selecting the appropriate stimulus from two different pairs of stimuli, with correct choices dependent on the initial sample stimulus encountered at the start of the maze. The important difference between this and previous tests of matching-to-sample is that the sample stimuli were not the same as the subsequent choice stimuli. Horizontal or vertical grating served as samples with colour and pattern stimuli presented at the choice stage as symbolic matches. Honeybees successfully learned this task.

Collett (2005) suggests that the mechanism for success on

Fig. 3. Bumblebees flew through a series of chambers $(35 \mathrm{~cm}$ diameter, $25 \mathrm{~cm}$ in height) in a delayed matching-to-sample task. The chambers were connected by tubes $10 \mathrm{~cm}$ long and $5 \mathrm{~cm}$ in diameter. Bees entered the sample chamber and viewed the sample stimulus (blue quadrilateral) before moving through an opening (gray circle) into the Choice chamber. Choice of the matching stimulus (blue or yellow quadrilateral) led to a sucrose reward in one of the Reward chambers. Choice of the non-matching stimulus was unrewarded (upper panel). Bumblebees did no better than chance on this task after many trials (lower panel).

used with honeybees and were unsuccessful. We trained seven bumblebees (B. impatiens) to fly through a Y-maze that consisted of a sample chamber, choice chamber, and reward chambers, each connected by a short tube (Fig. 3). Once bees were flying through the chamber each bee was given 80 trials of DMTS training with blue and yellow colour cues. There were four trial types, a yellow sample with a colour match on either the right or the left, and a blue sample with a colour match on either the right or the left. All trial types were presented equally and pseudorandomized. None of the bumblebees learned the task and performance did not differ from chance after 80 trials (Fig. 3). The colour stimuli were the same as those used in the reversal experiments described earlier in which bumblebees readily learned both discriminations and reversals. The number of trials and number of bees tested in this experiment were comparable to those of Giurfa et al. (2001) in which honeybees successfully learned a DMTS task, though it is worth noting that 80 trials is considerably fewer than the hundreds of trials often given to pigeons during DMTS training (Hogan et al., 1981).

Not all attempts to train honeybees on DMTS, however, have been successful. Brown et al. (1998) trained honeybees on a delayed matching and a delayed non-matching task using colour stimuli presented on a horizontally mounted computer screen. The honeybees showed a bias towards choosing the matching stimulus regardless of which stimulus - match or non-match - was rewarded. These results are consistent with the hypothesis that honeybees are successful at DMTS through use of their natural SDMTS may be route learning by sequential priming, using visual information from one location to prime memory for subsequent information. If sequence learning of this kind is the mechanism honeybees use in SDMTS, it is not clear why their performance differs from bumblebees. Bumblebees engage in traplining, which involves motor sequence learning (Thomson, 1996), and use visual information to guide motor output (Chittka, 1998). A systematic comparison of the performance of bumblebees and honeybees in DMTS and SDMTS tasks, examining the importance of winstay/lose-shift strategies and sequence priming for success on these tasks, would be very informative.

\section{Concept learning}

An early application of honeybees' success on the DMTS task was a test of concept learning. Giurfa et al. (2001) trained two groups of honeybees on either a colour or a grating DMTS task in the Ymaze and then tested the bees on unrewarded transfer trials to the other stimulus set. If the bees had used an associative mechanism to solve the DMTS task their performance with the novel stimuli should be at chance, but if they had used the concept of sameness to solve the problem originally, then application of that concept to novel stimuli would result in performance above chance. Bees trained on colour and transferred to gratings and those trained on gratings and transferred to colour both showed a preference for the matching choice. The authors ran additional experiments in which bees were required to learn the non-matching version of the task and then were given similar transfer tests. Bees again performed above chance suggesting that they had learned the concept of same/different. 
Concept learning has been studied in honeybees using other experimental paradigms, too. Avarguès-Weber et al. (2011) tested honeybees in a Y-maze on a discrimination between the concepts "above" and "below". The stimuli consisted of a target shape (e.g. a circle) placed either above or below a referent, a solid black horizontal bar. Bees were trained either to choose the stimulus representing the above relationship or the stimulus representing the below relationship. The bees were trained with a number of different target stimuli and then transferred successfully to novel stimuli. Importantly the bees' performance dropped to chance when the target stimulus was presented in the correct part of the visual field (either top or bottom depending on training), but the referent horizontal line was removed. Avarguès-Weber et al. (2012) expanded the task in order to look simultaneously at above/below and right/left relations. Honeybees successfully discriminated between these two relational configurations. Although there are many differences between "concepts" as they are understood in human cognition and the behaviour shown by bees (Chittka and Jensen, 2011), these two studies do provide persuasive demonstrations that bees are capable of learning relational "concepts" like above/below and left/right and support the previous findings with DMTS of Giurfa et al. (2001).

As with other research on cognitive processes in bees, there is much less known about concept learning in bumblebees. Brown and Sayde (2013) connected bumblebee colonies directly to a testing arena in which either two identical stimuli, or two dissimilar stimuli, were displayed on the floor. The stimuli consisted of either colours (yellow and blue) or gratings. There were detection zones within the arena that would open a door and provide reward if the bee moved to the correct detection zone given the stimuli presented (e.g. the right detection zone for stimuli that were the same), with correct detection zones counter balanced across colonies. The results showed that colonies made more correct than incorrect choices after training and the same was true when colonies were transferred to a novel set of stimuli. Data collection was automated and results were scored as the number of choices made. Because individual bee identities were not recorded, results were colonylevel choices. Patterns of choice by individual bumblebees were not known. The very different design of this study precludes direct comparison between bumblebees and honeybees, but this investigation of concept learning in bumblebees does suggest that bumblebees, at the colony level, are able to respond appropriately based on sameness or difference.

\section{Numerosity}

Exploration of number abilities in nonhuman animals has been extensive, numerosity having been demonstrated in species as distantly related as primates (Brannon and Terrace, 1998) and pigeons (Scarf et al., 2011). There are a number of studies showing that honeybees and bumblebees are capable of numerosity discriminations (Chittka and Geiger, 1995; Dacke and Srinivasan, 2008; Gross et al., 2009; Bar-Shai et al., 2011). Number is an apt topic for investigation in both bumblebees and honeybees because numerical information, such as the number of landmarks between the hive and a nectar source, could clearly assist in search for food and return to the hive (Pahl et al., 2013). The number of flowers in an inflorescence, the number of flowers visited in the current patch, the number of other individuals currently feeding at a nectar source, the number of eggs produced, the number of cells containing honey, pollen or brood are all numerical properties of the environment and potentially important to both bumblebees and honeybees.

The paradigms used to study number in bees draw heavily on naturalistic foraging tasks. Chittka and Geiger (1995) tested whether or not honeybees would use the number of landmarks to locate a feeder when landmark number and distance were confounded. Honeybees were trained to a feeder that was a specific number of landmarks and a specific distance away from their colony. After training, the bees were tested on variations of the task. The variations involved changing the distance between the landmarks and adding or reducing the number of landmarks. The results on test trials showed that bees predominantly used distance to guide their search for the feeder, but on some test trials a significant number of bees searched at the correct feeder according to landmark number. Dacke and Srinivasan (2008), building on the work of Chittka and Geiger (1995), trained honeybees to locate a feeder based on number of landmarks when distance was made uninformative. The honeybees successfully learned to locate the feeder based on sequentially encountered landmarks and even transferred to novel landmark stimuli. The behaviour of honeybees on these tasks is consistent with the view that number can serve as a distance cue for foraging bees.

Number judgments in naturalistic tasks have also been studied in bumblebees. Bar-Shai et al. (2011) observed bumblebees that foraged on a plant which produces exactly five nectaries in each flower, the bristly hollyhock $A$. setosa. If bumblebees were using number information while foraging they should probe the flowers no more than five times, because a sixth probe would usually be unrewarded. As predicted, bumblebees observed in the wild most frequently probed $A$. setosa 5 times during a visit. Following field observation the authors conducted a more controlled laboratory investigation of numerically determined flower departure. Bumblebees were trained that only two feeders in a patch would provide nectar, and that after two feeder visits the bumblebees would have to move to another patch in order to continue receiving rewards. This was intended to engage the same mechanism for departure as at an $A$. setosa flower. Throughout training the number of flowers visited by bees within a patch declined and approached the optimal number of two visits per patch. The results suggest that, similar to honeybees, bumblebees can use numerical information to aid foraging. It is worth noting that the bumblebees were trained for a very large number of trials, 200 , so while bumblebees are able to use numerical information, they may not learn it as easily as other cues. This result is similar to the way in which honeybees used numerical information in Chittka and Geiger's (1995) study. Numerical information was used, but distance was the preferred cue.

Not all investigations of numerical abilities in honeybees have used naturalistic paradigms; the DMTS task has also been used to test numerical abilities in honeybees (Gross et al., 2009). In a Ymaze honeybees successfully matched the number of items in a sample, up to the number four. This ability to accurately discriminate small numbers suggests the bees may be holding individual items in working memory, a so-called object file mechanism for determining number (Merritt et al., 2012). The object file system for numerical discrimination breaks down when more than 4 items are involved, even for humans. This contrasts with the approximate number system that can handle any number of items but obeys Weber's law, namely that the ratio between numbers determines whether or not they can be discriminated (Merritt et al., 2012). Honeybees' ability to match exact number rather than approximate magnitude up to the number four is very similar to exact number discrimination in monkeys (Hauser et al., 2000) and may indicate that a similar mechanism for discriminating small numbers is at work. Although there are not a great many studies on number sense in bees, there has been research on both bumblebees and honeybees in the wild and in different laboratory paradigms. The results are consistent in showing that both bumblebees and honeybees use numerosity to make foraging decisions. This result is what might be expected, given that both honeybees and bumblebees are likely to gain from using number in some form to estimate distances, 
assess foraging outcomes, and perhaps monitor reproduction and resources in the nest.

\section{Spatial cognition}

Social bees are ideal subjects for research on spatial cognition because they can be relied on to return to their colony after a foraging excursion. Like food storing birds that can be relied on to return to their food caches (Sherry and Hoshooley, 2007), bumblebees and honeybees must be able to discriminate among real world spatial locations and return to particular places, like the nest, from variable and unpredictable starting points.

\subsection{Foraging}

The foraging behaviour of bumblebees and honeybees has two components that involve spatial cognition: finding nectar or pollen sources and finding the nest or hive again following foraging. The latter has a memory component, since it consists of return to a familiar location. The former can sometimes, but not always, have a memory component because bees both return to familiar food sources and search for as-yet-undiscovered food sources. Honeybees can be guided by the waggle dance in their search for feeding sites that other members of the colony have already discovered, but honeybees also return to food sources they themselves have previously visited. Even with waggle dance information to assist them, honeybees have to remember the direction and distance information they have obtained from the waggle dance. Bumblebees do not follow directions they have obtained in the hive to locate food sources, though they may acquire some information about candidate food sources in the nest (Dornhaus and Chittka, 2001, 2004, 2005; Saleh and Chittka, 2006; Molet et al., 2009).

Naïve $B$. terrestris bumblebees begin foraging within a few days of emerging from pupae and show a transition within a few more days from arcing and looping orientation flights near the hive to long directed foraging trips (Osborne et al., 2013). Honeybees are older, around 14 days, when they begin foraging (Capaldi et al., 2000) and they, too, show initial orientation flights near the hive followed by longer foraging trips. Honeybees' orientation flights may be more restricted to the region around the hive compared to bumblebee orientation flights (Capaldi et al., 2000).

Honeybees use many navigational strategies to make the round trip from the hive to nectar sources, including dead reckoning, orientation to familiar landmarks near the hive, orientation to distant landmarks such as the panorama of the horizon, the sun compass, and skylight polarization (Pahl et al., 2011). Much less is known about bumblebee navigation.

\subsection{Homing}

The classic test of spatial ability is homing performance (Tinbergen, 1932; Perdeck, 1958). Jean-Henri Fabre made what were probably the first observations of homing ability in bees (Fabre, 1879, 1882). In modern research, Goulson and Stout (2001) found that bumblebees (B. terrestris) captured leaving the nest and displaced were able to home from up to $10 \mathrm{~km}$ away. Over a range of displacements from 1 to $15 \mathrm{~km}$, a significant negative relation was found between the proportion of bees returning home and displacement distance, falling to zero for displacements greater than $10 \mathrm{~km}$. Bumblebees could, however, take up to 9 days to return home even from an intermediate distance of $3.5 \mathrm{~km}$. Given the time taken to home, Goulson and Stout (2001) concluded that bumblebees are probably not using the sun compass or the earth's magnetic field to orient, but are instead searching until they encounter familiar visual landmarks. Because bumblebees were displaced rapidly in opaque boxes, compass information determined at the release site would not by itself provide enough information for successful homing. Bumblebees would also have to know where they were in relation to home. The behaviour of the bees suggests they could not determine this at the release site but instead searched until they encountered familiar landmarks. Interestingly, one bee was later found foraging at a release site over $4 \mathrm{~km}$ from the nest, indicating that the foraging range in $B$. terrestris may be substantial. Other bumblebees, including $B$. pascorum, $B$. rederatrius and $B$. muscorum are thought to forage much closer to home and displacement studies with these species could provide new information on species differences and the role of experience in long-distance homing (Goulson and Stout, 2001).

Displacement of honeybees had a similar outcome when bees were displaced in four compass directions (Pahl et al., 2011). Homing success declined with displacement distance over the range 0 to $13 \mathrm{~km}$, falling to zero at about $6 \mathrm{~km}$ in three out of four compass directions but only falling to zero at $11 \mathrm{~km}$ in the remaining compass direction. The horizon included distinctive elevation features when viewed from the different release points and the authors concluded that these visual features were the cause of differences in homing success from different compass directions. As with bumblebees, homing from novel release sites appeared to require successful detection of familiar landmarks rather than sun compass, skylight polarization or magnetic field information. Honeybees, like bumblebees in the experiment described above, were displaced in dark containers and so could not extract homing information simply from their displacement to the release sites. Because bees had been captured as they returned to the hive and then displaced, path integration could not account for successful homing either because their path integration mechanism would, presumably, be set to zero at the hive. As with bumblebees, successful homing sometimes took several days.

Both bumblebees and honeybees therefore appear to home from displacements well outside their normal foraging range by searching until they encounter familiar visual landmarks. The long times required in some cases for homing and the nature of the experimental procedures indicate that neither honeybees nor bumblebees are able to determine their location relative to home at unfamiliar release sites.

Honeybees on their first flights out of the hive and when departing from a new food source "turn back and look" (Lehrer, 1993). This behaviour consists of turning $180^{\circ}$ to face the hive or food source and re-approaching it repeatedly while swaying to and fro (Lehrer, 1993). The behaviour can initially last up to $20 \mathrm{~s}$ but decreases in duration on subsequent departures and then ceases. Bumblebees leaving their nest behave differently, performing a series of looping flights (Philippides et al., 2013). On the return approach to the nest, bumblebees fly in a series of zigzags in which the angle between flight direction and the nest, and the angle between body orientation and flight direction, recapitulate those of the departure flight. The result is that the retinal position of the nest and the retinal positions of landmarks near the nest closely match during departure and return flights. This suggests that bumblebees relocate their nest by matching their nest-centred view of the surroundings on approach to a remembered nest-centred view acquired during departure (Philippides et al., 2013).

Animals can orient to the geometry of the space in which they find themselves (Cheng, 1986). The test of orientation by geometry is that an animal should confuse locations that are geometrically equivalent, opposite corners of a rectangular enclosure, for example. Two of the corners of a rectangular enclosure will have a long wall on the left and a short wall on the right and thus be geometrically equivalent. Both bumblebees and honeybees searching for nectar reward can orient using the geometry of an enclosure and make the same error of confusing diagonally opposite and geometrically equivalent corners (Dittmar et al., 2014). Honeybees and 
bumblebees do not, however, confuse the geometrically equivalent corners when given additional featural cues either inside or outside the enclosure. When geometry and featural cues were placed in conflict, bees reliably chose the reward location based on featural cues not geometry. Bumblebees and honeybees behaved almost identically in these experiments (Dittmar et al., 2014).

\subsection{Non-foraging spatial behaviour}

Foraging is not the only context in which bees engage in spatial search. Male and female bumblebees and honeybees both search for mates. Bumblebee queens and honeybee workers search for suitable sites to establish colonies, and cuckoo bumblebees search for the nests of other species of bumblebee to parasitize. These behaviours do not involve memory for a familiar location like the nest or a previously visited food source. There may be a memory component, however, in structuring search to maximize encounter with mates or potential nest sites and to avoid revisits to places already searched. The paths of honeybees searching for their nest or a food source can be modelled as Lévy flights (Reynolds et al., 2013; Reynolds et al., 2007a,b). In Lévy flight, the distribution of the lengths of path segments follows the power law: short segments are common, intermediate length segments are less common, and very long segments also occur but with low probability. The arcing and looping orientation flights of bumblebees described earlier show the characteristics of Lévy flight (Osborne et al., 2013). It may not be surprising that both honeybees and bumblebees sometimes exhibit Lévy flight. Flights of this kind can produce optimal search (Reynolds et al., 2013). There is very little information, however, on the role of learning, memory or other cognitive processes in search or on whether bumblebees and honeybees use the same cognitive processes during search.

\section{Timing}

Bumblebees are able to estimate the duration of time intervals in the range of a few seconds up to several minutes both in a bumblebee-sized operant chamber and in a more natural foraging environment (Boisvert and Sherry, 2006; Boisvert et al., 2007). Interval timing of this kind is distinct from circadian timing which bumblebees and other insects also exhibit (Chittka et al., 2013; Pahl et al., 2007; Moore et al., 1998) and which plays an important role in time-place learning. In time-place learning, animals associate reward at a particular location with the time of day when reward occurs. Honeybees and stingless bees both show time-place learning (von Frisch, 1967; Murphy and Breed, 2008; de Jesus et al., 2014; Moore et al., 2011). Interval timing is quite a different phenomenon. In interval timing, animals time the duration of intervals much shorter than a day. In experiments with bumblebees, we made nectar available on a fixed interval (FI) schedule (Boisvert and Sherry, 2006). In such a schedule, the first response after a fixed interval of time has elapsed produces reward. Bumblebees' responses showed the pattern of responding typically found in vertebrates on an FI schedule, withholding responding for about one third to one half of the interval and exhibiting the maximum rate of responding at the end of the interval. Bumblebees were also able to time multiple FIs simultaneously (Boisvert and Sherry, 2006).

In a more natural foraging situation, bumblebees learned to suspend foraging on low value artificial flowers containing $2.5 \mu \mathrm{l}$ of $25 \%$ sucrose when a high value flower containing $40 \mu \mathrm{l}$ of $50 \%$ sucrose became available (Boisvert et al., 2007). Visits to the high value flower peaked at about the time the high value flower became available, which was either $30 \mathrm{~s}$ or $150 \mathrm{~s}$ after beginning foraging on the low value flowers. This evidence for interval timing ability is particularly interesting because bumblebees had to estimate a time interval that began with their own initiation of foraging on the low value flowers, not a learned signal indicating the beginning of the interval to be timed as is usual in most interval timing experiments.

Bumblebees may time their return to flowers according to the rates of nectar replenishment in the field, though there is little direct evidence for this. Most studies of trapline foraging in bumblebees focus on the spatial rather than the temporal patterns of traplining (Lihoreau et al., 2012). Whether honeybees are capable of interval timing remains to be determined, though there is evidence that honeybees are sensitive to durations. Honeybee foragers use the time needed to find a food-storing worker to take the nectar they have collected in order to adjust their foraging to the ability of the colony to process the nectar they collect (Seeley and Tovey, 1994).

\section{Metacognition}

Metacognition, monitoring one's own cognition (Roberts et al., 2012), is generally considered the domain of humans and primates, but has recently been explored in honeybees (Perry and Barron, 2013). The standard paradigm for testing metacognition involves presenting an animal with a test and then giving them the option of either taking or opting out of the test (Hampton, 2001). If the animal chooses to take the test they will receive a reward if they are correct and punishment if they are incorrect. If they opt out they do not get the reward, but they are able to avoid punishment. In this paradigm, animals should opt out when tests are hard and they are likely to receive punishment, but they should choose to take the test in order to get a reward when they have enough information to get the test right. There are a number of variations on the design, but the principle remains the same: animals that can monitor whether they have the appropriate information to succeed or fail on a particular trial should use the opt out choice when appropriate to maximize reward.

In Perry and Barron's (2013) task, honeybees were presented with a discrimination task and they could either take the test or opt out and be presented with another discrimination. Target stimuli were identical shapes presented either above or below a horizontal reference line. Choice of the target above the line was rewarded with $50 \mu \mathrm{l}$ of $2 \mathrm{M}$ sucrose; choice of the target below the line was punished with $50 \mu \mathrm{l}$ of $50 \mathrm{mM}$ quinine hydrochloride and a puff of air. This reward contingency was reversed for half of the subjects. Discrimination was made increasingly difficult by moving the target stimuli closer to the reference line until in the most difficult discrimination the targets overlapped the reference line and the two stimuli were indistinguishable. Bees were trained with one set of targets and tested with novel targets.

The results showed that some bees chose to opt out more when the discriminations were hard and that they performed better when they elected to take a test than when they were forced to take a test. The results are interesting, but the authors admit that they are consistent with other proposed associative learning explanations for success on metacognition tasks. Stimuli consisting of targets near or overlapping the reference line could become associated with a high probability of punishment, leading to avoidance of both stimuli and a choice to opt out on such trials.

Sensitivity to the ability to solve a problem is not without precedent in honeybees however. The natural turn back and look behaviour, mentioned earlier, that honeybees perform to gather navigational information is highly flexible and bees change the duration of their learning flights depending on their need for information (Wei et al., 2002). Turn back and look is different from what is generally considered metacognition (Roberts et al., 2012), but it does provide support for the idea that honeybees are sensitive to the amount of information they possess and when to gather more. 


\section{Conclusions}

Given the differences in social organization and foraging between bumblebees and honeybees, we might expect there to be differences in what they can learn and remember and how accurately they can remember it. From the evidence that is available, bumblebees seem to be better at serial reversal learning than honeybees. This indicates that bumblebees are more behaviourally flexible and can better adjust their behaviour to changing reward contingencies. This conforms to what might be expected if bumblebees, as individual foragers, are more likely to have to solve the problem of changing floral reward contingencies themselves, compared to honeybees that are guided by information about floral reward acquired in the hive. This is also consistent with the observation of greater floral constancy in honeybees. Subsequent experiments may, of course, show that honeybees are better at serial reversal learning than the present evidence suggests, but flexibility and reversal learning seem promising topics for further comparative research on bee cognition.

Bumblebees, at least in our lab, seem stubbornly unable to solve delayed matching-to-sample tasks. This may depend on how well our task conformed to bumblebee perceptual abilities and motivation but there does, nevertheless, seem to be a difference between the ease with which honeybees and bumblebees learn to match to a previously presented visual stimulus. In other contexts, bumblebees are capable of recognizing stimuli they have previously encountered and so the apparent difference in performance between honeybees and bumblebees on this simple task merits further investigation.

Honeybees are capable of learning relational concepts. It is not known whether individual bumblebees can also learn concepts like same/different or above/below. Observation of groups of bumblebees indicate they will make choices consistent with having learned a same/different concept and further examination of concept learning in both honeybees and bumblebees would be valuable.

The numerical abilities of bumblebees and honeybees, so far as they have been investigated, seem very similar. A fruitful avenue for research would be examination of natural foraging, orientation, and social behaviour in which number is the preferred dimension that bees attend to.

Whether bumblebees differ from honeybees in spatial cognition is an open question. Homing performance from unfamiliar release sites seems very similar in both species. There is, however, much less known about the details of bumblebee spatial cognition than is known for honeybees. In honeybees, landmark recognition, estimation of goal direction from landmarks, matching of the visual surround to visual landmarks, use of optic flow for visual "odometry" and many other topics have been extensively examined (Srinivasan, 2014; Cheng, 2000; Collett et al., 2006; Dittmar et al., 2011). Research on bumblebees has tended to focus on the outcomes of spatial ability, such as traplining. Much less is known about the mechanisms of bumblebee spatial cognition. A trip to collect nectar and return to the nest is, in its simplest form, identical in an experienced bumblebee or honeybee: leave a familiar central place, fly to a familiar patch of flowers, and return. The simple prediction is that the spatial abilities used to solve this problem are identical in bumblebees and honeybees. Testing this prediction might uncover unexpected differences in how these closely related animals perform spatial tasks and produce new information on the variety of ways in which such spatial problems can be solved.

Bumblebees are able to time interval durations up to $150 \mathrm{~s}$ and probably longer. There is no reason to suppose honeybees do not have similar timing abilities but this has never been tested. As with spatial cognition, a direct comparison of timing abilities in bumblebees and honeybees could be very informative. It is not known how bumblebees use their timing ability. The durations that have been examined experimentally are short compared to the nectar replenishment times of flowers, but the timing ability of bumblebees for much longer durations not been tested. Timing may also be used in other contexts, such as handling times for extracting floral nectar, estimating the time required to off-load nectar (Seeley and Tovey, 1994), or integration over time of visual flow (Srinivasan et al., 2000). Differences in timing ability between bumblebees and honeybees might lead to new discoveries about the role of time in bumblebee and honeybee foraging, social behaviour, and navigation.

Honeybees seem capable of assessing whether or not they possess sufficient information to make a correct choice in a foraging task. They also seem capable of determining when their turn back and look behaviour has gathered enough information to successfully return to a spatial location. Memory monitoring of this kind, or metacognition, may not be restricted to vertebrates but instead be a widespread ability of animals that make choices on the basis of previous experience. Further examination of metacognition and a comparison or bumblebee and honeybee memory monitoring may lead to new discoveries about a little-understood phenomenon in animal cognition.

There have been a small number of studies in which the cognitive abilities and behaviour of bumblebees and honeybees have been explicitly compared under standardized conditions (Townsend-Mehler et al., 2011; Townsend-Mehler and Dyer, 2012; Dittmar et al., 2014). These studies stand as clear examples of what can be learned from well-designed comparative research with bumblebees and honeybees. Ideally, information on the cognitive abilities of many more species from a much broader range of families and tribes would make it possible to use phylogenetic comparative methods to examine the evolution of the cognitive traits of bees. Given the practical difficulty of such an enterprise and the confounding effects of perception, attention, and motivation in comparing cognition in different species, a comparative approach based on neural circuitry and patterns in gene expression might hold more promise (Chittka et al., 2012). It is clear, however, that many challenging questions about animal cognition can be fruitfully examined in bees and that the comparative approach has shown variation in how cognitive tasks are solved in nature. There is, in addition, a further important way in which research with bumblebees and honeybees can make a significant contribution to the contemporary study of animal cognition.

Comparative cognition as a field has become increasingly tangled up in questions that may be unresolvable. Do animals have episodic memory for events they have experienced? Can they plan for the future? Do animals mentally travel backward and forward in time, re-experiencing the past and pre-experiencing the future? Do animals possess a theory of mind or consciousness? Questions such as these, found as one researcher has put it in the "romantic" regions of the animal cognition landscape where folk psychology replaces a rationalist and materialist approach (Penn, 2011), tend not to intrude into research on bees. To be sure, a few researchers have proposed that bees solve problems by conscious thought. In a discussion of the honeybee dance language, Griffin and Speck (2004) wrote, “... increasing evidence of complex cognition indicates that some invertebrates may take advantage of the effectiveness of conscious thinking when faced with challenging problems (p.13)." These authors take the complexity of honeybee behaviour and the fact that bees communicate as evidence for consciousness. For most researchers, neither complexity of behaviour nor communication provide evidence of consciousness and even for humans, the explanatory power of consciousness in understanding the causal processes of cognition is not clear (Shettleworth, 2010a,b). Most researchers working with bees are less inclined to invoke consciousness to understand how bumblebee and honeybee cognition works and instead focus on what kind of experience 
and neural processes cause behaviour. Research with bumblebees and honeybees may help lead research in comparative cognition away from its current preoccupation with anthropocentric issues and focus instead on ideas and hypotheses about cognition that can be tested by experiment and observation of animal behaviour. We think Jerry Hogan would approve.

\section{Acknowledgements}

We would like to thank Lars Chittka, Ken Cheng, and Graham Thompson for their many helpful comments on the manuscript. Preparation of this paper was supported by a Discovery Grant 105542 to DFS from the Natural Sciences and Engineering Research Council of Canada.

\section{References}

Avarguès-Weber, A., Chittka, L., 2014. Observational conditioning in flower choice copying by bumblebees (Bombus terrestris): influence of observer distance and demonstrator movement. PLoS One 9, e88415, http://dx.doi.org/10.1371/ journal.pone.0088415.

Avarguès-Weber, A., Dyer, A.G., Combe, M., Giurfa, M., 2012. Simultaneous mastering of two abstract concepts by the miniature brain of bees. Proc. Natl. Acad. Sci. U.S.A. 109, 7481-7486.

Avarguès-Weber, A., Dyer, A.G., Giurfa, M., 2011. Conceptualization of above and below relationships by an insect. Proc. R. Soc. London, Ser. B: Biol. Sci. 278, 898-905.

Bar-Shai, N., Keasar, T., Shmida, A., 2011. The use of numerical information by bees in foraging tasks. Behav. Ecol. 22, 317-325.

Bitterman, M.E., 1969. Habit-reversal and probability learning: rats, birds and fish. In: Gilbert, R.M., Sutherland, N.S. (Eds.), Animal Discrimination Learning. Academic Press, New York, NY.

Bitterman, M.E., Menzel, R., Fietz, A., Schäfer, S., 1983. Classical conditioning of proboscis extension in honeybees (Apis mellifera). J. Comp. Psychol. 97, 107-119.

Blough, D.S., 1959. Delayed matching in the pigeon. J. Exp. Anal. Behav. 2, 151-160.

Boisvert, M.J., Sherry, D.F., 2006. Interval timing by an invertebrate, the bumble bee Bombus impatiens. Curr. Biol. 16, 1-5.

Boisvert, M.J., Veal, A.J., Sherry, D.F., 2007. Floral reward production is timed by an insect pollinator. Proc. R. Soc. London, Ser. B: Biol. Sci. 274, 1831-1937.

Brannon, E.M., Terrace, H.S., 1998. Ordering of the numerosities 1 to 9 by monkeys. Science 282, 746-749.

Brown, M.F., McKeon, D., Curley, T., Weston, B., Lambert, C., Lebowitz, B., 1998 Working memory for color in honeybees. Anim. Learn. Behav. 26, 264-271.

Brown, M.F., Sayde, J.M., 2013. Same/different discrimination by bumblebee colonies. Anim. Cogn. 16, 117-125.

Capaldi, E.A., Smith, A.D., Osborne, J.L., Fahrbach, S.E., Farris, S.M., Reynolds, D.R., Edwards, A.S., Martin, A., Robinson, G.E., Poppy, G.M., Riley, J.R., 2000. Ontogeny of orientation flight in the honeybee revealed by harmonic radar. Nature 403 , 537-540.

Cardinal, S., Danforth, B.N., 2011. The antiquity and evolutionary history of social behavior in bees. PLoS One 6, e21086.

Cardinal, S., Straka, J., Danforth, B.N., 2010. Comprehensive phylogeny of apid bees reveals the evolutionary origins and antiquity of cleptoparasitism. Proc. Natl. Acad. Sci. U.S.A. 107, 16207-16211.

Cheng, K., 1986. A purely geometric module in the rat's spatial representation. Cognition 23, 149-178.

Cheng, K., 2000. How honyebees find a place: lessons from a simple mind. Anim. Learn. Behav. 28, 1-15.

Chittka, L., 1998. Sensorimotor learning in bumblebees: long-term retention and reversal training. J. Exp. Biol. 201, 515-524.

Chittka, L., Geiger, K., 1995. Can honeybees count landmarks? Anim. Behav. 49, 159-164.

Chittka, L., Jensen, K., 2011. Animal cognition: concepts from apes to bees. Curr. Biol. 21, R116-R119.

Chittka, L., Rossiter, S.J., Skorupski, P., Fernando, C., 2012. What is comparable in comparative cognition? Philos. Trans. R. Soc. London, Ser. B: Biol. Sci.367, 2677-2685.

Chittka, L., Stelzer, R.J., Stanewsky, R., 2013. Daily changes in ultraviolet light levels can synchronize the circadian clock of bumblebees (Bombus terrestris). Chronobiol. Int. 30, 434-442.

Chittka, L., Thomson, J.D., Waser, N.M., 1999. Flower constancy, insect psychology, and plant evolution. Naturwissenschaften, 86.

Collett, T.S., 2005. Invertebrate memory: honeybees with a sense of déjà vu. Curr. Biol. 15, R419-R421.

Collett, T.S., Graham, P., Harris, R.A., de-Ibarra, N.H., 2006. Navigational memories in ants and bees: memory retrieval when selecting and following routes. Adv. Study Behav. 36, 123-172.

Couvillon, M.J., 2012. The dance legacy of Karl von Frisch. Insectes Soc. 59, 297-306.

Couvillon, P.A., Bitterman, M.E., 1986. Performance of honeybees in reversal and ambiguous-cue problems: tests of a choice model. Anim. Learn. Behav. 14, 225-231.
Dacke, M., Srinivasan, M.V., 2008. Evidence for counting in insects. Anim. Cogn. 11, 683-689.

Danforth, B.N., Cardinal, S., Praz, C., Almeida, E.A.B., Michez, D., 2013. The impact of molecular data on our understanding of bee phylogeny and evolution. Annu. Rev. Entomol. 58, 57-78.

Darwin, C., 1841. Humble-bees. The Gardeners' Chronicle No. 34, 550.

Davey, G., 1989. Ecological Learning Theory. Routledge, New York, NY.

Dawson, E.H., Chittka, L., 2014. Bumblebees (Bombus terrestris) use social information as an indicator of safety in dangerous environments. Proc. R. Soc. London, Ser. B: Biol. Sci. 281, 20133174, 10.1098/rspb.2013.3174.

de Jesus, T.N.C.S., Venturieri, G.C., Contrera, F.A.L., 2014. Time-place learning in the bee Melipona fasciculata (Apidae, Meliponini). Apidologie 45, 257-265.

Dittmar, L., Egelhaaf, M., Stürlz, W., Boeddeker, N., 2011. The behavioral relevance of landmark texture for honeybee homing. Front. Behav. Neurosci. 5, http://dx.doi.org/10.3389/fnbeh.2011.00020.

Dittmar, L., Stürzl, W., Jetzschke, S., Mertes, M., Boeddeker, N., 2014. Out of the box: how bees orient in an ambiguous environment. Anim. Behav. 89, 13-21.

Dornhaus, A., Chittka, L., 2001. Food alert in bumblebees (Bombus terrestris): possible mechanisms and evolutionary implications. Behav. Ecol. Sociobiol. 50, 570-576.

Dornhaus, A., Chittka, L., 2004. Information flow and regulation of foraging activity in bumble bees (Bombus spp.). Apidologie 35, 183-192.

Dornhaus, A., Chittka, L., 2005. Bumble bees (Bombus terrestris) store both food and information in honeypots. Behav. Ecol. 16, 661-666.

Dyer, A.G., Dorin, A., Reinhardt, V., Garcia, J.E., Rosa, M.P.G., 2014. Bee reverselearning behavior and intra-colony differences: simulations based on behaviora experiments reveal benefits of diversity. Ecol. Modell. 277, 119-131.

Dyer, A.G., Spaethe, J., Prack, S., 2008. Comparative psychophysics of bumblebee and honeybee colour discrimination and object detection. J. Comp. Physiol. A 194, 617-627.

Evangelista, C., Kraft, P., Dacke, M., Labhart, Srinivasan, M.V., 2014. Honeybee navigation: critically examining the role of the polarization compass. Philos. Trans. R. Soc. London, Ser. B: Biol. Sci. 369, 20130037.

Fabre, J.-H., 1879. Souvenirs entomologique Études sur l'instinct et les moeurs des insectes. Ch. Delagrave, Paris.

Fabre, J.-H., 1882. Nouveax souvenirs entomologique Études sur l'instinct et les moeurs des insectes Paris.

Free, J.B., 1963. The flower constancy of honeybees. J. Anim. Ecol. 32, 119-131.

Free, J.B., 1970. The flower constancy of bumblebees. J. Anim. Ecol. 39, 395-402.

Giurfa, M., Sandoz, J.-C., 2012. Invertebrate learning and memory: fifty years of olfactory conditioning of the proboscis extension response in honeybees. Learn. Mem. 19, 54-66.

Giurfa, M., Zhang, S., Jenett, A., Menzel, R., Srinivasan, M.V., 2001. The concepts of 'sameness' and 'difference' in an insect. Nature 410, 930-933.

Goulson, D., 2009. Bumblebees: Behaviour, Ecology and Conservation. Oxford University Press, Oxford, UK.

Goulson, D., Park, K.J., Tinsley, M.C., Bussière, L.F., Vallejo-Marin, M., 2013. Social learning drives handedness in nectar-robbing bumblebees. Behav. Ecol. Sociobiol. 67, 1141-1150.

Goulson, D., Stout, J.C., 2001. Homing ability of the bumblebee Bombus terrestris (Hymeoptera: Apidae). Apidologie 32, 105-111.

Griffin, D.R., Speck, G.B., 2004. New evidence of animal consciousness. Anim. Cogn. 7, 5-18.

Gross, H.J., Pahl, M., Si, A., Zhu, H., Tautz, J., Zhang, S., 2009. Number-based visual generalization in the honeybee. PLoS One 4, e4263.

Grüter, G., Farina, W.M., 2009. The honeybee waggle dance: can we follow the steps? Trends Ecol. Evol. 24, 242-247.

Hampton, R.R., 2001. Rhesus monkeys know when they remember. Proc. Natl. Acad. Sci. U.S.A. 98, 5359-5362.

Hauser, M.D., Carey, S., Hauser, L., 2000. Spontaneous number representation in semi-free-ranging rhesus monkeys. Proc. R. Soc. London, Ser. B: Biol. Sci. 267, 829-833.

Hedtke, S.M., Patiny, S., Danforth, B.N., 2013. The bee tree of life: a supermatrix approach to apoid phylogeny and biogeography. BMC Evol. Biol. 13, 138.

Heinrich, B., 1979. Bumblebee Economics. Harvard University Press, Harvard, MA.

Heinrich, B., Mudge, P.R., Deringis, P.G., 1977. Laboratory analysis of flower constancy in foraging bumblebees: Bombus ternaries and B. terricola. Behav. Ecol. Sociobiol. 2, 247-265.

Hogan, D.E., Edwards, C.A., Zentall, T.R., 1981. Delayed matching in the pigeon: interference produced by the prior delayed matching trial. Anim. Learn. Behav. 9 395-400.

Hogan, J.A., 1994. The concept of cause in the study of behaviour. In: Hogan, J.A. Bolhuis, J.J. (Eds.), Causal Mechanisms of Behavioral Development. Cambridge University Press, Cambridge.

Hogan, J.A., 2014. A framework for the study of behavior. Behav. Processes (in press)

Hogan, J.A., Bolhuis, J.J., 2009. Tinbergen's four questions and contemporary behavioral biology. In: Bolhuis, J.J., Verhulst, S. (Eds.), Tinbergen's Legacy: Function and Mechanism in Behavioral Biology. Cambridge University Press, Cambridge.

Jandt, J.M., Dornhaus, A., 2009. Spatial organization and division of labour in the bumblebee Bombus impatiens. Anim. Behav. 77, 641-651.

Kawakita, A., Ascher, J.S., Sota, T., Kato, M., Roubick, D.W., 2008. Phylogenetic analysis of the corbiculate bee tribes based on 12 nuclear protein-coding genes (Hymenoptera: Apoidea: Apidae). Apidologie 39, 163-175.

Kearns, C.A., Thomson, J.D., 2001. The Natural History of Bumblebees: A Sourcebook for Investigators. University of Colorado Press, Boulder, CO.

Komischke, B., Giurfa, M., Lachnit, H., Malun, D., 2002. Successive olfactory reversa learning in honeybees. Learn. Mem. 9, 122-129. 
Laverty, T.M., Plowright, R.C., 1988. Flower handling by bumblebees: a comparison of specialists and generalists. Anim. Behav. 36, 733-740

Leadbeater, E., Chittka, L., 2005. A new mode of information transfer in foraging bumblebees? Curr. Biol. 15, R447-R448, http://dx.doi.org/10.1016/ j.cub.2005.06.011.

Leadbeater, E., Chittka, L., 2008. Social transmission of nectar-robbing behaviour in bumble-bees. Proc. R. Soc. London, Ser. B: Biol. Sci., http://dx.doi.org/10.1098/ rspb.2008.0270 (First Cite e-publishing Online Date April 22, 2008).

Lehrer, M., 1993. Why do bees turn back and look? J. Comp. Physiol. A 172, 549-563.

Leonhardt, S.D., Blüthgen, N., 2012. The same, but different: pollen foraging in honeybee and bumblebee colonies. Apidologie 43, 449-464.

Lihoreau, M., Chittka, L., Raine, N.E., 2010. Travel optimization by foraging bumblebees through readjustments of traplines after discovery of new feeding locations. Am. Nat. 176, 744-757.

Lihoreau, M., Raine, N.E., Reynolds, M.R., Stelzer, R.J., Lim, K.S., Smith, A.D., Osborne, J.L., Chittka, L., 2012. Radar tracking and motion-sensitive cameras on flowers reveal the development of pollinator multi-destination routes over large spatial scales. PLoS Biol. 10, e1001392.

Menzel, R., 2012. The honeybee as a model for understanding the basis of cognition. Nat. Rev. Neurosci. 13, 758-768.

Merritt, D.J., DeWind, N.K., Brannon, E.M., 2012. Comparative cognition of number representation. In: Zentall, T.R., Wasserman, E.A. (Eds.), Oxford Handbook of Comparative Cognition. Oxford University Press, Oxford.

Michener, C.D., 2007. Bees of the World. Johns Hopkins University Press, Baltimore, MD.

Molet, M., Chittka, L., Raine, N.E., 2009. How floral odours are learned inside the bumblebee (Bombus terretris) nest. Naturwissenschaften 96, 213-219.

Moore, D., Moore, J.E., Cheeseman, I.M., Fahrbach, S.E., Robinson, G.E., 1998. Timekeeping in the honeybee colony: integration of circadian rhythms and division of labour. Behav. Ecol. Sociobiol. 43, 147-160.

Moore, D., Van Nest, B.N., Seier, E., 2011. Diminishing returns: the influence of experience and environment on time-memory extinction in honey bee foragers. J. Comp. Physiol. A 197, 641-651.

Morawetz, L., Spaethe, J., 2012. Visual attention in a complex search task differs between honeybees and bumblebees. J. Exp. Biol. 215, 2515-2523.

Mota, T., Giurfa, M., 2010. Multiple reversal olfactory learning in honeybees. Front. Behav. Neurosci. 4, 48

Murphy, C.M., Breed, M.D., 2008. Time-place learning in a neotropical stingless bee, Trigona fulviventris Guérin (Hymenoptera: Apidae). J. Kansas Entomol. Soc. 81, $73-76$.

Osborne, J.L., Smith, A., Clark, S.J., Reynolds, D.R., Barron, M.C., Lim, K.S., Reynolds, D.R., 2013. The ontogeny of bumblebee flight trajectories: from naïve explorers to experienced foragers. PLoS One 8, e78681.

Pahl, M., Si, A., Zhang, S., 2013. Numerical cognition in bees and other insects. Front Psychol. 4, 162 .

Pahl, M., Zhu, H., Pix, W., Tautz, J., Zhang, S., 2007. Circadian timed episodic-like memory-a bee knows what to do when, and also where. J. Exp. Biol. 210 3559-3567.

Pahl, M., Zhu, H., Tautz, J., Zhang, S., 2011. Large scale homing in honeybees. PLoS One 6, e19669.

Penn, D.C., 2011. How folk psychology ruined comparative psychology and how scrub jays can save it. In: Menzel, R., Fischer, J. (Eds.), Animal Thinking: Contemporary Issues in Comparative Cognition. MIT Press, Cambridge, MA.

Perdeck, A.C., 1958. Two types of orientation in migrating starlings, Sturnus vulgaris L., and chaffinches, Fringilla coelebs L., as revealed by displacement experiments. Ardea 46, 1-37.

Perry, C.J., Barron, A.B., 2013. Honey bees selectively avoid difficult choices. Proc. Natl. Acad. Sci. U.S.A. 110, 19155-19159.

Perry, C.J., Barron, A.B., Cheng, K., 2013. Invertebrate learning and cognition: relating phenomena to neural substrate. WIREs Cogn. Sci., http://dx.doi.org/10.1002/wcs.1248.

Philippides, A., de Ibarra, N.H., Riabinina, O., Collett, T.S., 2013. Bumblebee calligraphy: the design and control of flight motifs in the learning and return flights of Bombus terrestris. J. Exp. Biol. 216, 1093-1104.

Raine, N.E., Chittka, L., 2012. No trade-off between learning speed and associative flexibility in bumblebees: a reversal learning test of multiple colonies. PLoS One 7. e45096, http://dx.doi.org/10.1371/journal.pone.0045096.

Raine, N.E., Ings, T.C., Dornhaus, A., Nehal, S., Chittka, L., 2006. Adaptation, genetic drift, pleiotroy, and history in the evolution of bee foraging behavior. Adv. Study Behav. 36, 305-354.

Reynolds, A.M., Schultheiss, P., Cheng, K., 2013. Are Lévy flight patterns derived from the Weber-Fechner law in distance estimation? Behav. Ecol. Sociobiol. $67,1219-1226$
Reynolds, A.M., Smith, A.D., Menzel, R., Greggers, U., Reynolds, D.R., Riley, J.R., 2007a. Displaced honey bees perform opitmal scale-free search flights. Ecology 88, 1955-1961.

Reynolds, A.M., Smith, A.D., Reynolds, D.R., Carreck, N.L., Osborne, J.L., 2007b. Honeybees perform optimal scale-free searching flights when attempting to locate a food source. J. Exp. Biol. 210, 3763-3770.

Roberts, W.A., 1998. Principles of Animal Cognition. McGraw Hill, Boston, MA

Roberts, W.A., McMillan, N., Musolino, E., Cole, M., 2012. Information seeking in animals: metacognition? Comp. Cogn. Behav. Rev. 7, 85-109.

Romanes, G.J., 1884. Mental evolution in animals. Appleton, New York, NY.

Saleh, N., Chittka, L., 2006. The importance of experience in the interpretation of conspecific chemical signals. Behav. Ecol. Sociobiol. 61, 215-220.

Scarf, D., Hayne, H., Colombo, M., 2011. Pigeons on par with primates in numerica competence. Science 334, 1664.

Seeley, T., Tovey, D.C.A., 1994. Why search time to find a food-storer accurately indicates the relative rates of nectar collecting and nectar processing in honey bee colonies. Anim. Behav. 47, 311-316.

Seeley, T.D., 1985. Honeybee Ecology: A Study of Adaptation in Social Life. Princeton University Press, Princeton, NJ.

Sherry, D.F., Hoshooley, J.S., 2007. Neurobiology of spatial behavior. In: Otter, K.A (Ed.), The Ecology and Behavior of Chickadees and Titmice: An Integrated Approach. Oxford University Press, Oxford.

Shettleworth, S.J., 1998. Cognition, evolution, and behavior. Oxford University Press, New York, NY.

Shettleworth, S.J., 2010a. Clever animals and killjoy explanations in comparative psychology. Trends Cogn. Sci. 14, 477-481.

Shettleworth, S.J., 2010b. Cognition Evolution and Behavior. Oxford University Press, Oxford.

Shettleworth, S.J., 2013. Fundamentals of Comparative Cognition. Oxford University Press, Oxford, UK

Spaethe, E., Weidenmüller, A., 2002. Size variation and foraging rate in bumblebees (Bombus terrestris). Insectes Soc. 49, 142-146.

Srinivasan, M.V., 2011. Honeybees as a model for the study of visually guided flight, navigation, and biologically inspired robotics. Physiol. Rev. 91, 413-460.

Srinivasan, M.V., 2014. Going with the flow: a brief history of the study of the honeybee's navigational odometer. J. Comp. Physiol. A 200, 563-573.

Srinivasan, M.V., Zhang, S., Altwein, M., Tautz, J., 2000. Honeybee navigation: nature and calibration of the odometer. Science 287, 851-853.

Strang, C.G., Sherry, D.F., 2014. Serial reversal learning in bumblebees (Bombus impatiens). Anim. Cogn. 17, 723-734

Thompson, G.J., Oldroyd, B.P., 2004. Evaluating alternative hypotheses for the origin of eusociality in cobiculate bees. Mol. Phylogenet. Evol. 33, 452-456.

Thomson, J.D., 1996. Trapline foraging by bumblebees: I. Persistence of flight-path geometry. Behav. Ecol. 7, 158-164.

Tinbergen, N., 1932. Über die Orientierung des Bienenwolfes (Philanthus triangulum Fabr.). Z. Vgl. Physiol. 16, 305-334

Townsend-Mehler, J.M., Dyer, F.C., 2012. An integrated look at decision-making in bees as they abandon a depeleted food source. Behav. Ecol. Sociobiol. 66 275-286.

Townsend-Mehler, J.M., Dyer, F.C., Maida, K., 2011. Deciding when to explore and when to persist: a comparison of honeybees and bumblebees in their response to downshifts in reward. Behav. Ecol. Sociobiol. 65, 305-312.

von Frisch, K., 1967. The Dance Language and Orientation of Bees. Bellnap Press Harvard, Cambridge, MA.

von Helversen, O., 1974. Das experiment: Dressurversuche mit bienen. Biol. Unsere Zeit 4, 119-124

Wei, C.A., Rafalko, S.L., Dyer, F.C., 2002. Deciding to learn: modulation of learning flights in honeybees, Apis mellifera. J. Comp. Physiol. A 188, 725-737.

Worden, B.D., Papaj, D.R., 2005. Flower choice copying in bumblebees. Biol. Lett. 1 504-507, http://dx.doi.org/10.1098/rsbl.2005.0368.

Wright, A.A., 1997. Concept learning and learning strategies. Psychol. Sci. 8, 119-123.

Zentall, T.R., Hogan, E., 1978. Same/different concept learning in the pigeon: the effect of negative instances and prior adaptation to transfer stimuli. J. Exp. Anal. Behav. 30, 177-186.

Zentall, T.R., Wasserman, E.A., 2012. The Oxford Handbook of Comparative Cognition. Oxford University Press, Oxford, UK.

Zhang, S., Bock, F. Tautz, J., Srinivasan, M.V., 2005. Visual working memory in decision making by honeybees. Proc. Natl. Acad. Sci. U.S.A. 102, 5250-5255.

Zhang, S., Lehrer, M., Srinivasan, M.V., 1999. Honeybee memory: navigation by associative grouping and recall of visual stimuli. Neurobiol. Learn. Mem. 72, 180-201.

Zhang, S., Pahl, M., 2012. Visually guided decision making in foraging honeybees Front. Neurosci. 6, 88 